\title{
On generalized intuitionistic fuzzy subhyperalgebras of Boolean hyperalgebras
}

\author{
Kostaq Hila ${ }^{*}$, Serkan Onar², Bayram Ali Ersoy ${ }^{2}$ and Bijan Davvaz ${ }^{3}$
}

\section{"Correspondence:}

kostaq_hila@yahoo.com

'Department of Mathematics \&

Computer Science, Faculty of

Natural Sciences, University of

Gjirokastra, Gjirokastra, Albania

Full list of author information is

available at the end of the article

\begin{abstract}
This paper deals with a special class of hyperalgebra, called Boolean hyperalgebra, which is redefined in it. We introduce the concepts of generalized intuitionistic fuzzy subhyperalgebras and generalized intuitionistic fuzzy hyperideals of Boolean hyperalgebras. A necessary and sufficient condition for an intuitionistic fuzzy subset of the Boolean hyperalgebra to be a generalized intuitionistic fuzzy subhyperalgebra (hyperideal) is proved. Images and inverse-images of generalized intuitionistic fuzzy subhyperalgebra (hyperideal) under Boolean hyperalgebra homomorphism are studied.

MSC: Primary 03E72; secondary 08A72

Keywords: Boolean hyperalgebra; generalized intuitionistic fuzzy subhyperalgebra; generalized intuitionistic fuzzy hyperideal; generalized intuitionistic fuzzy quotient Boolean hyperalgebra
\end{abstract}

\section{Introduction}

The applications of mathematics in other disciplines, for example, in informatics, play a key role, and they have represented, in the last decades, one of the purpose of the study of the experts of hyperstructure theory all over the world. Hyperstructure theory was introduced in 1934 by a French mathematician Marty [1], at the 8th Congress of Scandinavian Mathematicians, where he defined hypergroups based on the notion of hyperoperation, began to analyze their properties and applied them to groups. In the following decades and nowadays, a number of different hyperstructures have been widely studied from the theoretical point of view and for their applications to many subjects of pure and applied mathematics by many mathematicians such as in fuzzy sets and rough set theory, optimization theory, theory of discrete event dynamical systems, cryptography, codes, analysis of computer programs, automata, formal language theory, combinatorics, artificial intelligence, probability, graphs and hypergraphs, geometry, lattices and binary relations. In a classical algebraic structure, the composition of two elements is an element, while in an algebraic hyperstructure, the composition of two elements is a set. Several books have been written on hyperstructure theory, see [2-5]. A recent book on hyperstructures [3] points out their applications in rough set theory, cryptography, codes, automata, probability, geometry, lattices, binary relations, graphs and hypergraphs. Another book [4] is devoted especially to the study of hyperring theory. Several kinds of hyperrings are introduced and analyzed. The volume ends with an outline of applications in chemistry and

○2013 Hila et al.; licensee Springer. This is an Open Access article distributed under the terms of the Creative Commons Attribution License (http://creativecommons.org/licenses/by/2.0), which permits unrestricted use, distribution, and reproduction in any medium, provided the original work is properly cited. 
physics, analyzing several special kinds of hyperstructures: $e$-hyperstructures and transposition hypergroups.

An algebraic hyperstructure (henceforth simply called a hyperalgebra) is a set endowed with one or more hyperoperations, i.e., multi-valued operations mapping a pair of elements to a set of elements. Hyperalgebras are generalizations of classical algebras, e.g., hypergroup is a generalization of group [2], hyperlattice and superlattice are generalizations of lattice [6-8] and so on.

As it is well known, in 1965 Zadeh [9] introduced the notion of set $\mu$ on a non-empty set $X$ as a function from $X$ to the unite real interval $I=[0,1]$ as a fuzzy set. In 1971, Rosenfeld [10] introduced fuzzy sets in the context of group theory and formulated the concept of a fuzzy subgroup of a group. Since then, many researchers have been engaged in extending the concepts of abstract algebra to the framework of the fuzzy setting (cf. [11-16]). The study of fuzzy hyperstructure is an interesting research topic of fuzzy sets and it is applied to the theory of algebraic hyperstructure. Fuzzy hyperalgebras were introduced rather recently. The relations between fuzzy sets and hyperstructures have been already considered by Corsini, Davvaz, Zahedi, Leoreanu-Fotea, Ameri, Borzoei, Hasankhani, Tofan, Kehagias, and many others (see $[17,18]$ ). All these works are based on the use of fuzzy hyperoperations, i.e., a fuzzy hyperalgebra is a set equipped with one or more fuzzy hyperoperations. The concept of fuzzy hyperoperation is a natural extension of crisp hyperoperation: as already mentioned, a crisp hyperoperation maps a pair of elements to a crisp subset of elements; a fuzzy hyperoperation maps a pair of elements to a fuzzy subset. But in fuzzy sets theory, there is no means to incorporate the hesitation or uncertainty in the membership degrees. As an important generalization of the notion of fuzzy sets on a nonempty set $X$, in 1983, Atanassov introduced in $[19,20]$ the concept of intuitionistic fuzzy sets on a non-empty set $X$ which give both a membership degree and a non-membership degree. The only constraint on these two degrees is that the sum must be smaller than or equal to 1 . The concept has been applied to various algebraic structures $(c f .[21,22])$. Since then, the notion of intuitionistic fuzzy sets has been explored by researchers, and a number of theoretical and practical results have appeared. Intuitionistic fuzzy sets as a generalization of fuzzy sets can be useful in situations when description of a problem by a (fuzzy) linguistic variable, given in terms of a membership function only, seems too rough. For example, in decision-making problems, particularly in the case of medial diagnosis, sales analysis, new product marketing, financial services, etc., there is a fair chance of the existence of a non-null hesitation part at each moment of evaluation of an unknown object. The relations between intuitionistic fuzzy sets and algebraic hyperstructures have been already considered by many mathematicians such as Davvaz, Dudek, Jun, Corsini, Zhan, Leoreanu-Fotea etc. (cf. [23-26]). In [27-31], some relations between fuzzy sets and Boolean algebras are considered.

In this paper, using Atanassov's idea, we introduce the concepts of generalized intuitionistic fuzzy subhyperalgebras and generalized intuitionistic fuzzy hyperideals of Boolean hyperalgebras. A necessary and sufficient condition for an intuitionistic fuzzy subset of a Boolean hyperalgebra to be a generalized intuitionistic fuzzy subhyperalgebra (hyperideal) is proved. Images and inverse-images of the generalized intuitionistic fuzzy subhyperalgebra (hyperideal) under Boolean hyperalgebra homomorphism are studied. We introduce the notion of generalized intuitionistic fuzzy quotient Boolean hyperalgebra and several properties of it are studied. 


\section{Preliminaries}

Recall first the basic terms and definitions from the hyperstructure theory and fuzzy set theory.

A map $\circ: H \times H \rightarrow \mathcal{P}^{*}(H)$ is called hyperoperation or join operation on the set $H$, where $H$ is a non-empty set and $\mathcal{P}^{*}(H)=\mathcal{P}(H) \backslash\{\emptyset\}$ denotes the set of all non-empty subsets of $H$.

If $x \in H$ and $A, B$ are non-empty subsets of $H$, then

$$
A \circ B=\bigcup_{a \in A, b \in B} a \circ b, \quad A \circ x=A \circ\{x\}, \quad \text { and } \quad x \circ B=\{x\} \circ B .
$$

In general, for a positive integer $n$, an $n$-ary hyperoperation $\beta$ on $H$ is a function $\beta$ : $H^{n} \rightarrow P^{*}(H)$. We say that $n$ is the arity of $\beta$. A subset $S$ of $H$ is closed under the $n$-ary hyperoperation $\beta$ if $\left(x_{1}, \ldots, x_{n}\right) \in S^{n}$ implies that $\beta\left(x_{1}, \ldots, x_{n}\right) \subseteq S$. A nullary hyperoperation on $H$ is just an element of $P^{*}(H)$; i.e., a non-void subset of $H$. A hyperalgebraic system or a hyperalgebra $\left\langle H,\left(\beta_{i}: i \in I\right)\right\rangle$ is the set $H$ with a collection $\left(\beta_{i} \mid i \in I\right)$ of hyperoperations on $H$.

A subset $S$ of a hyperalgebra $\mathbb{H}=\left\langle H,\left(\beta_{i}: i \in I\right)\right\rangle$ is a subhyperalgebra of $H$ if $S$ is closed under each hyperoperation $\beta_{i}$ for all $i \in I$, that is, $\beta_{i}\left(a_{1}, \ldots, a_{n_{i}}\right) \subseteq S$, whenever $\left(a_{1}, \ldots, a_{n_{i}}\right) \in S^{n_{i}}$.

Definition 2.1 Let $X$ be a non-empty set. A fuzzy subset $\mu$ of $X$ is a function $\mu: X \rightarrow[0,1]$. Let $\mu, \lambda$ be two fuzzy subsets of $X$, we say that $\mu$ is contained in $\lambda$ if $\mu(x) \leq \lambda(x), \forall x \in X$.

Definition 2.2 Let $\mu$ be a fuzzy subset of a set $X$, and let $t \in[0,1]$. The set $\mu_{t}=\{x \in X$ : $\mu(x) \geq t\}$ is called a level subset of $\mu$.

Definition 2.3 Let $\mathbb{H}=\left\langle H,\left(\beta_{i}: i \in I\right)\right\rangle$ and $\overline{\mathbb{H}}=\left\langle H,\left(\overline{\beta_{i}}: i \in I\right)\right\rangle$ be two similar hyperalgebras. A map $h$ from $\mathbb{H}$ into $\overline{\mathbb{H}}$ is called

(i) a homomorphism if for every $i \in I$ and all $\left(a_{1}, \ldots, a_{n_{i}}\right) \in H^{n_{i}}$, we have that $h\left(\beta_{i}\left(a_{1}, \ldots, a_{n_{i}}\right)\right) \subseteq \overline{\beta_{i}}\left(h\left(a_{1}\right), \ldots, h\left(a_{n_{i}}\right)\right)$;

(ii) a good homomorphism if for every $i \in I$ and all $\left(a_{1}, \ldots, a_{n_{i}}\right) \in H^{n_{i}}$, we have that $h\left(\beta_{i}\left(a_{1}, \ldots, a_{n_{i}}\right)\right)=\overline{\beta_{i}}\left(h\left(a_{1}\right), \ldots, h\left(a_{n_{i}}\right)\right)$.

Let us introduce below Boolean hyperalgebra in a different way (see [32], cf. [33-36]).

Definition 2.4 A Boolean hyperalgebra is a hyperstructure $\left(B, \vee, \wedge, 0,1,^{\prime}\right)$, where $B$ is a non-empty set, $\vee: B \times B \rightarrow \mathcal{P}(B), \wedge: B \times B \rightarrow \mathcal{P}(B),{ }^{\prime}: B \rightarrow B, 0$ and 1 are two distinguished elements such that:

(1) $\forall a \in B, a \in a \vee a, a \in a \wedge a$;

(2) $\forall a, b \in B, a \vee b=b \vee a, a \wedge b=b \wedge a$;

(3) $\forall a, b, c \in B, a \vee(b \vee c)=(a \vee b) \vee c, a \wedge(b \wedge c)=(a \wedge b) \wedge c$;

(4) $\forall a, b \in B, a \in a \vee(a \wedge b), a \in a \wedge(a \vee b)$;

(5) $\forall a, b \in B, a \in a \vee b \Rightarrow b \in a \wedge b$;

(6) $\forall a, b, c \in B, a \wedge(b \vee c)=(a \wedge b) \vee(a \wedge c)$;

(7) $\forall a \in B, 0 \vee a=a, 0 \wedge a=0,1 \in 1 \vee a, 1 \wedge a=a$;

(8) $\forall a \in B, a \vee a^{\prime}=1, a \wedge a^{\prime}=0$.

A Boolean hyperalgebra is said to be strong if

(9) $\forall a, b \in B, a \vee b=\left\{w \in B: a^{\prime} \wedge w^{\prime}=b^{\prime} \wedge w^{\prime}=a^{\prime} \wedge b^{\prime}\right\}$. 
Definition 2.5 Let $\left(B, \vee, \wedge, 0,1,^{\prime}\right)$ be a Boolean hyperalgebra. If for any $a, b \in B, b \in a \vee b$, then $a$ is not larger than $b$, and we use $a \leq b$ to stand for.

\section{Generalized intuitionistic fuzzy subhyperalgebras}

In this section, we introduce the notion of generalized intuitionistic fuzzy subhyperalgebras of a Boolean hyperalgebra and several properties of them are investigated. Several conditions of an intuitionistic fuzzy subset of a Boolean hyperalgebra to be a generalized intuitionistic fuzzy subhyperalgebra are given.

Atanassov introduced in $[19,37]$ the concept of intuitionistic fuzzy sets defined on a non-empty set $X$ as objects having the form

$$
A=\left\{\left\langle x, \mu_{A}(x), \lambda_{A}(x)|| x \in X\right\}\right.
$$

where the functions $\mu_{A}: X \rightarrow[0,1]$ and $\lambda_{A}: X \rightarrow[0,1]$ denote the degree of membership (namely $\mu_{A}(x)$ ) and the degree of non-membership (namely $\lambda_{A}(x)$ ) of each element $x \in X$ to the set $A$ respectively, and $0 \leq \mu_{A}(x)+\lambda_{A}(x) \leq 1$ for all $x \in X$.

Obviously, any ordinary fuzzy set may be written as

$$
A=\left\{\left\langle x, \mu_{A}(x), 1-\mu_{A}(x)|| x \in X\right\} .\right.
$$

Let $A$ and $B$ be two intuitionistic fuzzy sets on $X$. The following expressions are defined in $[37,38]$.

(1) $A \subseteq B$ if and only if $\mu_{A}(x) \leq \mu_{B}(x)$ and $\lambda_{A}(x) \geq \lambda_{B}(x)$ for all $x \in X$.

(2) $A=B$ if and only if $A \subseteq B$ and $B \subseteq A$.

(3) $A^{c}=\left\{\left\langle x, \lambda_{A}(x), \mu_{A}(x)\right\rangle \mid x \in X\right\}$.

(4) $A \cap B=\left\{\left\langle x, \mu_{A}(x) \wedge \mu_{B}(x), \lambda_{A}(x) \vee \lambda_{B}(x)\right\rangle \mid x \in X\right\}$.

(5) $A \cup B=\left\{\left\langle x, \mu_{A}(x) \vee \mu_{B}(x), \lambda_{A}(x) \wedge \lambda_{B}(x)\right\rangle \mid x \in X\right\}$.

(6) $\square A=\left\{\left\langle x, \mu_{A}(x), 1-\mu_{A}(x)\right\rangle \mid x \in X\right\}$.

(7) $\diamond A=\left\{\left\langle x, 1-\lambda_{A}(x), \lambda_{A}(x)\right\rangle \mid x \in X\right\}$.

For the sake of simplicity, we use the symbol $A=\left(\mu_{A}, \lambda_{A}\right)$ for an intuitionistic fuzzy set $A=\left\{\left\langle x, \mu_{A}(x), \lambda_{A}(x)\right\rangle \mid x \in X\right\}$.

For any fuzzy set $\mu$ of $X$ and any $t \in[0,1], U(\mu ; t)=\{x \in X \mid \mu(x) \geq x\}$ is called an upper bound t-level cut of $\mu$ and $L(\mu ; t)=\{x \in X \mid \mu(x) \leq x\}$ is called a lower bound t-level cut of $\mu$.

Let $f$ be a mapping from a set $X$ to a set $Y$. Let $A=\left(\mu_{A}, \lambda_{A}\right)$ be an intuitionistic fuzzy subset of $X$ and $B=\left(\mu_{B}, \lambda_{B}\right)$ be an intuitionistic fuzzy subset of $Y$. Then the inverse image $f^{-1}(B)$ of $B$ is the intuitionistic fuzzy set of $X$ defined by $f^{-1}(B)=\left(f^{-1}\left(\mu_{B}\right), f^{-1}\left(\lambda_{B}\right)\right)$, where $f^{-1}\left(\mu_{B}\right)(x)=\mu_{B}(f(x))$ and $f^{-1}\left(\lambda_{B}\right)=\lambda_{B}(f(x))$. The image $f(\mu)$ of $\mu$ is the fuzzy subset of $Y$ defined by

$$
f(\mu)(y)= \begin{cases}\sup \left\{\mu(t) \mid t \in f^{-1}(y)\right\} & \text { if } f^{-1}(y) \neq \emptyset \\ 0 & \text { otherwise }\end{cases}
$$

for all $y \in Y$. 
The image $f(B)$ of the intuitionistic fuzzy subset $B=\left(\mu_{B}, \lambda_{B}\right)$ is the intuitionistic fuzzy set in $Y$ defined by $f(B)=\left(f\left(\mu_{B}\right), f^{*}\left(\lambda_{B}\right)\right)$, where for every $y \in Y$,

$$
\begin{aligned}
& f\left(\mu_{B}\right)(y)= \begin{cases}\sup \left\{\mu_{B}(t) \mid t \in f^{-1}(y)\right\} & \text { if } f^{-1}(y) \neq \emptyset, \\
0 & \text { otherwise, }\end{cases} \\
& f^{*}\left(\lambda_{B}\right)(y)= \begin{cases}\inf \left\{\lambda_{B}(t) \mid t \in f^{-1}(y)\right\} & \text { if } f^{-1}(y) \neq \emptyset, \\
0 & \text { otherwise. }\end{cases}
\end{aligned}
$$

Definition 3.1 A fuzzy subset $\mu$ of a Boolean hyperalgebra $B$ is said to be a fuzzy subhyperalgebra of $B$ if for all $a, b \in B$,

(1) $\inf _{t \in a \vee b} \mu(t) \geq \min \{\mu(a), \mu(b)\}$;

(2) $\inf _{t \in a \wedge b} \mu(t) \geq \min \{\mu(a), \mu(b)\}$;

(3) $\mu\left(a^{\prime}\right) \geq \mu(a)$.

Definition 3.2 A fuzzy subset $\mu$ of a Boolean hyperalgebra $B$ is said to be a fuzzy hyperideal of $B$ if for all $a, b \in A$,

(1) $\inf _{t \in a \vee b} \mu(t) \geq \min \{\mu(a), \mu(b)\}$;

(2) $\inf _{t \in a \wedge b} \mu(t) \geq \mu(b)$.

Definition 3.3 Let $B$ be a Boolean hyperalgebra and $t \in(0,1]$. An intuitionistic fuzzy subset $A=\left(\mu_{A}, \lambda_{A}\right)$ of $B$ is said to be a generalized intuitionistic fuzzy subhyperalgebra of $B$ if for all $a, b \in B$,

(1) $\inf _{x \in a \vee b} \mu_{A}(x) \geq \min \left\{\mu_{A}(a), \mu_{A}(b), t\right\}$ and $\sup _{x \in a \vee b} \lambda_{A}(x) \leq \max \left\{\lambda_{A}(a), \lambda_{A}(b), t\right\}$;

(2) $\inf _{x \in a \wedge b} \mu_{A}(x) \geq \min \left\{\mu_{A}(a), \mu_{A}(b), t\right\}$ and $\sup _{x \in a \wedge b} \lambda_{A}(x) \leq \max \left\{\lambda_{A}(a), \lambda_{A}(b), t\right\}$;

(3) $\mu_{A}\left(a^{\prime}\right) \geq \min \left\{\mu_{A}(a), t\right\}$ and $\lambda_{A}\left(a^{\prime}\right) \leq \max \left\{\lambda_{A}(a), t\right\}$.

It is obvious that $H$ is the subhyperalgebra of $A$ iff the characteristic function $\chi_{H}$ of $H$ is a generalized intuitionistic fuzzy subhyperalgebra of $A$.

Proposition 3.4 Let $A=\left(\mu_{A}, \lambda_{A}\right)$ be an intuitionistic fuzzy subset of a Boolean hyperalgebra $B$ and $t \in(0,1]$. If for all $a, b \in B$, the following hold:

(1) $\inf _{x \in a \vee b} \mu_{A}(x) \geq \min \left\{\mu_{A}(a), \mu_{A}(b), t\right\}$ and $\sup _{x \in a \vee b} \lambda_{A}(x) \leq \max \left\{\lambda_{A}(a), \lambda_{A}(b), t\right\}$;

(2) $\mu_{A}\left(a^{\prime}\right) \geq \min \left\{\mu_{A}(a)\right.$,t $\}$ and $\lambda_{A}\left(a^{\prime}\right) \leq \max \left\{\lambda_{A}(a), t\right\}$,

then $A=\left(\mu_{A}, \lambda_{A}\right)$ is a generalized intuitionistic fuzzy subhyperalgebra of $B$.

Proof We have to prove only that condition (2) of Definition 3.3 holds. Indeed, for all $a, b \in B$, we have

$$
\begin{aligned}
\inf _{x \in a \wedge b} \mu_{A}(x) & =\inf _{y \in\left(a^{\prime} \vee b^{\prime}\right)^{\prime}} \mu_{A}(y) \\
& \geq \min \left\{\inf _{z \in\left(a^{\prime} \vee b^{\prime}\right)} \mu_{A}(z), t\right\} \\
& \geq \min \left\{\min \left\{\mu_{A}\left(a^{\prime}\right), \mu_{A}\left(b^{\prime}\right), t\right\}, t\right\} \\
& \geq \min \left\{\mu_{A}(a), \mu_{A}(b), t\right\},
\end{aligned}
$$


and

$$
\begin{aligned}
\sup _{x \in a \wedge b} \lambda_{A}(x) & =\sup _{y \in\left(a^{\prime} \vee b^{\prime}\right)^{\prime}} \lambda_{A}(y) \leq \max \left\{\sup _{z \in\left(a^{\prime} \vee b^{\prime}\right)} \lambda_{A}(z), t\right\} \\
& \leq \max \left\{\max \left\{\lambda_{A}\left(a^{\prime}\right), \lambda_{A}\left(b^{\prime}\right), t\right\}, t\right\} \\
& \leq \max \left\{\lambda_{A}(a), \lambda_{A}(b), t\right\} .
\end{aligned}
$$

So, condition (2) of Definition 3.3 is true.

Proposition 3.5 Let $A=\left(\mu_{A}, \lambda_{A}\right)$ be an intuitionistic fuzzy subset of a Boolean hyperalgebra $B$ and $t \in(0,1]$. If for all $a, b \in B$, the following hold:

(1) $\inf _{x \in a \wedge b} \mu_{A}(x) \geq \min \left\{\mu_{A}(a), \mu_{A}(b), t\right\}$ and $\sup _{x \in a \wedge b} \lambda_{A}(x) \leq \max \left\{\lambda_{A}(a), \lambda_{A}(b), t\right\}$;

(2) $\mu_{A}\left(a^{\prime}\right) \geq \min \left\{\mu_{A}(a), t\right\}$ and $\lambda_{A}\left(a^{\prime}\right) \leq \max \left\{\lambda_{A}(a), t\right\}$,

then $A=\left(\mu_{A}, \lambda_{A}\right)$ is a generalized intuitionistic fuzzy subhyperalgebra of $B$.

Proof We have to prove only that condition (1) of Definition 3.3 holds. Indeed, for all $a, b \in B$, we have

$$
\begin{aligned}
\inf _{x \in a \vee b} \mu_{A}(x) & =\inf _{y \in\left(a^{\prime} \wedge b^{\prime}\right)^{\prime}} \mu_{A}(y) \geq \min \left\{\inf _{z \in a^{\prime} \wedge b^{\prime}} \mu_{A}(z), t\right\} \\
& \geq \min \left\{\min \left\{\mu_{A}\left(a^{\prime}\right), \mu_{A}\left(b^{\prime}\right), t\right\}, t\right\} \\
& \geq \min \left\{\mu_{A}(a), \mu_{A}(b), t\right\}
\end{aligned}
$$

and

$$
\begin{aligned}
\sup _{x \in a \vee b} \lambda_{A}(x) & =\sup _{y \in\left(a^{\prime} \wedge b^{\prime}\right)^{\prime}} \lambda_{A}(y) \leq \max \left\{\sup _{z \in a^{\prime} \wedge b^{\prime}} \lambda_{A}(z), t\right\} \\
& \leq \max \left\{\max \left\{\lambda_{A}\left(a^{\prime}\right), \lambda_{A}\left(b^{\prime}\right), t\right\}, t\right\} \\
& \leq \max \left\{\lambda_{A}(a), \lambda_{A}(b), t\right\} .
\end{aligned}
$$

So, condition (1) of Definition 3.3 is true.

Proposition 3.6 Let $A=\left(\mu_{A}, \lambda_{A}\right)$ be an intuitionistic fuzzy subset of a Boolean hyperalgebra $B$. Then $A=\left(\mu_{A}, \lambda_{A}\right)$ is a generalized intuitionistic fuzzy subhyperalgebra of $B$ if and only if $U\left(\mu_{A} ; s\right)$ and $L\left(\lambda_{A} ; s\right)$ are subhyperalgebras of $B$ for all $s \in(0, t]$ when they are nonempty.

Proof Let $A=\left(\mu_{A}, \lambda_{A}\right)$ be a generalized intuitionistic fuzzy subhyperalgebra of $B, s \in(0, t]$ and $a, b \in U\left(\mu_{A} ; s\right)$. Then we have

$$
\begin{aligned}
& \inf _{x \in a \vee b} \mu_{A}(x) \geq \min \left\{\mu_{A}(a), \mu_{A}(b), t\right\} \geq s, \\
& \inf _{y \in a \wedge b} \mu_{A}(y) \geq \min \left\{\mu_{A}(a), \mu_{A}(b), t\right\} \geq s,
\end{aligned}
$$

and

$$
\mu_{A}\left(a^{\prime}\right) \geq \min \left\{\mu_{A}(a), t\right\} \geq s .
$$


So, $a \vee b \subseteq U\left(\mu_{A} ; s\right), a \wedge b \subseteq U\left(\mu_{A} ; s\right)$ and $a^{\prime} \in U\left(\mu_{A} ; s\right)$. Therefore $U\left(\mu_{A} ; s\right)$ is a subhyperalgebra of $B$. In a similar way, it is shown that $L\left(\lambda_{A} ; s\right)$ is a subhyperalgebra of $B$.

Conversely, let $U\left(\mu_{A} ; s\right)$ and $L\left(\lambda_{A} ; s\right)$ be subhyperalgebras of $B$ for all $s \in(0, t]$. If there exist $a, b \in B$ such that

$$
\inf _{x \in a \vee b} \mu_{A}(x)<c=\min \left\{\mu_{A}(a), \mu_{A}(b), t\right\}
$$

then $a, b \in U\left(\mu_{A} ; c\right), c \in(0, t]$ and $\inf _{y \in a \vee b} \mu_{A}(y)<c$. Since $U\left(\mu_{A} ; c\right)$ is a subhyperalgebra of $B$, we have $a \vee b \subseteq U\left(\mu_{A} ; c\right)$ and $\inf _{x \in a \vee b} \mu_{A}(x) \geq c$. This is impossible, because $\inf _{x \in a \vee b} \mu_{A}(x)<c$. Therefore, for all $a, b \in B, \inf _{x \in a \vee b} \mu_{A}(x) \geq \min \left\{\mu_{A}(a), \mu_{A}(b), t\right\}$.

Using the same method, for all $a, b \in B$, we have $\inf _{x \in a \wedge b} \mu_{A}(x) \geq \min \left\{\mu_{A}(a), \mu_{A}(b), t\right\}$ and $\mu_{A}\left(a^{\prime}\right) \geq \min \left\{\mu_{A}(a), t\right\}$.

In a similar way, we can operate for $L\left(\lambda_{A} ; s\right)$.

From all the above, we conclude that $A=\left(\mu_{A}, \lambda_{A}\right)$ is a generalized intuitionistic fuzzy subhyperalgebra of $B$.

Proposition 3.7 Let $B$ be a Boolean hyperalgebra and $A=\left(\mu_{A}, \lambda_{A}\right), C=\left(\mu_{C}, \lambda_{C}\right)$ be generalized intuitionistic fuzzy subhyperalgebras of $B$. Then $A \cap C$ is also a generalized intuitionistic fuzzy subhyperalgebra of B.

Proof Let $D=A \cap C$. For all $a, b \in B$, we have

$$
\begin{aligned}
\inf _{x \in a \vee b} \mu_{D}(x) & =\min \left\{\inf _{y \in a \vee b} \mu_{A}(y), \inf _{z \in a \vee b} \mu_{C}(z)\right\} \\
& \geq \min \left\{\min \left\{\mu_{A}(a), \mu_{A}(b), t\right\}, \min \left\{\mu_{C}(a), \mu_{C}(b), t\right\}\right\} \\
& \geq \min \left\{\min \left\{\mu_{A}(a), \mu_{C}(a)\right\}, \min \left\{\mu_{A}(b), \mu_{C}(b)\right\}, t\right\} \\
& \geq \min \left\{\mu_{D}(a), \mu_{D}(b), t\right\}
\end{aligned}
$$

and

$$
\begin{aligned}
\sup _{x \in a \vee b} \lambda_{D}(x) & =\max \left\{\sup _{y \in a \vee b} \lambda_{A}(y), \sup _{z \in a \vee b} \lambda_{C}(z)\right\} \\
& \leq \max \left\{\max \left\{\lambda_{A}(a), \lambda_{A}(b), t\right\}, \max \left\{\lambda_{C}(a), \lambda_{C}(b), t\right\}\right\} \\
& \leq \max \left\{\max \left\{\lambda_{A}(a), \lambda_{C}(a)\right\}, \max \left\{\lambda_{A}(b), \lambda_{C}(b)\right\}, t\right\} \\
& \leq \max \left\{\lambda_{D}(a), \lambda_{D}(b), t\right\} .
\end{aligned}
$$

Also,

$$
\left(\mu_{D}\right)\left(a^{\prime}\right) \geq \min \left\{\mu_{A}\left(a^{\prime}\right), \mu_{C}\left(a^{\prime}\right), t\right\} \quad \text { and } \quad\left(\lambda_{D}\right)\left(a^{\prime}\right) \leq \max \left\{\lambda_{A}\left(a^{\prime}\right), \lambda_{C}\left(a^{\prime}\right), t\right\} .
$$

Therefore $A \cap C$ is also a generalized intuitionistic fuzzy subhyperalgebra of $B$.

Proposition 3.8 Let $B, B^{\prime}$ be two Boolean hyperalgebras and $f: B \rightarrow B^{\prime}$ be a homomorphism. Iff is surjective and $A=\left(\mu_{A}, \lambda_{A}\right)$ is a generalized intuitionistic fuzzy subhyperalgebra of $B$, then $f(A)$ is a generalized intuitionistic fuzzy subhyperalgebra of $B^{\prime}$. 
Proof It is straightforward.

Proposition 3.9 Let $B, B^{\prime}$ be two Boolean hyperalgebras and $f: B \rightarrow B^{\prime}$ be a homomorphism. If $C=\left(\mu_{C}, \lambda_{C}\right)$ is a generalized intuitionistic fuzzy subhyperalgebra of $B^{\prime}$, then $f^{-1}(C)$ is a generalized intuitionistic fuzzy subhyperalgebra of $B$.

Proof It is straightforward.

\section{Generalized intuitionistic fuzzy hyperideals}

In this section, we introduce the notion of generalized intuitionistic fuzzy hyperideals of a Boolean hyperalgebra and several properties of them are investigated. Several conditions of an intuitionistic fuzzy subset of a Boolean hyperalgebra to be a generalized intuitionistic fuzzy hyperideal are given.

Definition 4.1 Let $B$ be a Boolean hyperalgebra, $t \in(0,1]$ and $A=\left(\mu_{A}, \lambda_{A}\right)$ be an intuitionistic fuzzy subset of $B$. Then $A$ is called a generalized intuitionistic fuzzy hyperideal of $B$ if for all $a, b \in B$, we have

(1) $\inf _{x \in a \vee b} \mu_{A}(x) \geq \min \left\{\mu_{A}(a), \mu_{A}(b), t\right\}$ and $\sup _{x \in a \vee b} \lambda_{A}(x) \leq \max \left\{\lambda_{A}(a), \lambda_{A}(b), t\right\}$,

(2) $\inf _{y \in a \wedge b} \mu_{A}(y) \geq \min \left\{\mu_{A}(b), t\right\}$ and $\sup _{y \in a \wedge b} \lambda_{A}(y) \leq \max \left\{\lambda_{A}(b), t\right\}$.

Proposition 4.2 Let $B$ be a Boolean hyperalgebra and $A=\left(\mu_{A}, \lambda_{A}\right)$ be an intuitionistic fuzzy subset of $B$. Then $A$ is a generalized intuitionistic fuzzy hyperideal of $B$ if and only if $U\left(\mu_{A} ; s\right)$ and $L\left(\lambda_{A} ; s\right)$ are hyperideals of $B$ for all $s \in(0, t]$ when they are non-empty.

Proof It is straightforward.

Proposition 4.3 Let $B$ be a Boolean hyperalgebra and $A=\left(\mu_{A}, \lambda_{A}\right), C=\left(\mu_{C}, \lambda_{C}\right)$ be two generalized intuitionistic fuzzy hyperideals of $B$. Then $A \cap C$ is also a generalized intuitionistic fuzzy hyperideal of $B$.

Proof It is straightforward.

Proposition 4.4 Let $B, B^{\prime}$ be two Boolean hyperalgebras and $f: B \rightarrow B^{\prime}$ be a homomorphism. Iff is surjective and $A=\left(\mu_{A}, \lambda_{A}\right)$ is a generalized intuitionistic fuzzy hyperideal of $B$, then $f(A)$ is a generalized intuitionistic fuzzy hyperideal of $B^{\prime}$.

Proof It is straightforward.

Proposition 4.5 Let $B, B^{\prime}$ be two Boolean hyperalgebras and $f: B \rightarrow B^{\prime}$ be a homomorphism. If $C=\left(\mu_{C}, \lambda_{C}\right)$ is a generalized intuitionistic fuzzy hyperideal of $B^{\prime}$, then $f^{-1}(C)$ is a generalized intuitionistic fuzzy hyperideal of $B$.

Proof It is straightforward.

Let $A=\left(\mu_{A}, \lambda_{A}\right)$ be a generalized intuitionistic fuzzy hyperideal of $A$. When $\mu_{A}(0)<t$ and $\lambda_{A}(0)<t$, the intuitionistic fuzzy hyperideal of $B$ is equal to the generalized intuitionistic fuzzy hyperideal of $B$. In the following, it is assumed that the generalized intuitionistic fuzzy hyperideal $A=\left(\mu_{A}, \lambda_{A}\right)$ of $B$ satisfies the conditions $\mu_{A}(0) \geq t$ and $\lambda_{A}(0) \leq t$. 
Proposition 4.6 Let $A$ be a Boolean hyperalgebra and $A=\left(\mu_{A}, \lambda_{A}\right)$ be a generalized intuitionistic fuzzy hyperideal of $B$. If $a, b \in B$ and $a \leq b$, then $\mu_{A}(a) \geq \min \left\{\mu_{A}(b), t\right\}$ and $\lambda_{A}(a) \leq \max \left\{\lambda_{A}(b), t\right\}$.

Proof We have $\mu_{A}(a) \geq \inf _{x \in a \wedge b} \mu_{A}(x) \geq \min \left\{\mu_{A}(b), t\right\}$ and $\lambda_{A}(a) \leq \sup _{x \in a \wedge b} \lambda_{A}(x) \leq$ $\max \left\{\lambda_{A}(b), t\right\}$.

Definition 4.7 Let $B$ be a Boolean hyperalgebra and $A=\left(\mu_{A}, \lambda_{A}\right)$ be a generalized intuitionistic fuzzy hyperideal of $B$. If there exists $a \in B$ such that $\mu_{A}(a)<t$ and $\lambda_{A}(a)<t$, then $A=\left(\mu_{A}, \lambda_{A}\right)$ is said to be a generalized intuitionistic fuzzy proper hyperideal of $B$.

Proposition 4.8 Let $B$ be a Boolean hyperalgebra and $A=\left(\mu_{A}, \lambda_{A}\right)$ be a generalized intuitionistic fuzzy hyperideal of $B$. Then $\mu_{A}(1)<t$ and $\lambda_{A}(1)<t$.

\section{Generalized intuitionistic fuzzy quotient Boolean hyperalgebra}

In this section, we introduce the notion of generalized intuitionistic fuzzy quotient Boolean hyperalgebras and several properties of them are studied.

Let $A=\left(\mu_{A}, \lambda_{A}\right)$ be a generalized intuitionistic fuzzy hyperideal of $B$, and let the intuitionistic fuzzy subset $[a]_{A}=\left(\mu_{[a]_{A}}, \lambda_{[a]_{A}}\right)$ of $B$ be defined as follows:

$$
\begin{aligned}
& \forall x \in B, \quad \mu_{[a]_{A}}(x)=\min \left\{\inf _{y \in\left(\left(a^{\prime} \wedge x\right) \vee\left(a \wedge x^{\prime}\right)\right)} \mu_{A}(y), t\right\} \quad \text { and } \\
& \lambda_{[a]_{A}}(x)=\max \left\{\sup _{y \in\left(\left(a^{\prime} \wedge x\right) \vee\left(a \wedge x^{\prime}\right)\right)} \lambda_{A}(y), t\right\} .
\end{aligned}
$$

We denote $B / A=\left\{[a]_{A}: a \in B\right\}$.

Proposition 5.1 Let $B$ be a Boolean hyperalgebra, $A=\left(\mu_{A}, \lambda_{A}\right)$ be a generalized intuitionistic fuzzy hyperideal of $B$ and $[a]_{A},[b]_{A} \in B / A$. Then we have $[a]_{A}=[b]_{A}$ iff $\inf _{y \in\left(\left(a^{\prime} \wedge b\right) \vee\left(a \wedge b^{\prime}\right)\right)} \mu_{A}(y) \geq t$ and $\sup _{y \in\left(\left(a^{\prime} \wedge b\right) \vee\left(a \wedge b^{\prime}\right)\right)} \lambda_{A}(y) \leq t$.

Proof Let $[a]_{A}=[b]_{A}$, then we have

$$
\min \left\{\inf _{y \in\left(\left(a^{\prime} \wedge b\right) \vee\left(a \wedge b^{\prime}\right)\right)} \mu_{A}(y), t\right\}=\min \left\{\inf _{z \in\left(\left(b^{\prime} \wedge b\right) \vee\left(b \wedge b^{\prime}\right)\right)} \mu_{A}(z), t\right\}=\min \left\{\mu_{A}(0), t\right\}=t
$$

Therefore $\inf _{y \in\left(\left(a^{\prime} \wedge b\right) \vee\left(a \wedge b^{\prime}\right)\right)} \mu_{A}(y) \geq t$. Also,

$$
\begin{aligned}
\max \left\{\sup _{y \in\left(\left(a^{\prime} \wedge b\right) \vee\left(a \wedge b^{\prime}\right)\right)} \lambda_{A}(y), t\right\} & =\max \left\{\sup _{z \in\left(\left(b^{\prime} \wedge b\right) \vee\left(b \wedge b^{\prime}\right)\right)} \lambda_{A}(z), t\right\} \\
& =\max \left\{\lambda_{A}(0), t\right\}=t .
\end{aligned}
$$

Therefore $\sup _{y \in\left(\left(a^{\prime} \wedge b\right) \vee\left(a \wedge b^{\prime}\right)\right)} \lambda_{A}(y) \leq t$.

On the other hand, for all $x \in B$,

$$
\begin{aligned}
\mu_{[a]_{A}}(x) & =\min \left\{\inf _{y \in\left(\left(a^{\prime} \wedge x\right) \vee\left(a \wedge x^{\prime}\right)\right)} \mu_{A}(y), t\right\} \\
& =\min \left\{\inf _{y \in\left(\left(\left(a^{\prime} \wedge x\right) \vee\left(a \wedge x^{\prime}\right)\right) \wedge\left(b \vee b^{\prime}\right)\right)} \mu_{A}(y), t\right\}
\end{aligned}
$$




$$
\begin{aligned}
& =\min \left\{\inf _{y \in\left(\left(a^{\prime} \wedge b \wedge x\right) \vee\left(a \wedge b^{\prime} \wedge x^{\prime}\right) \vee\left(a^{\prime} \wedge b^{\prime} \wedge x\right) \vee\left(a \wedge b \wedge x^{\prime}\right)\right)} \mu_{A}(y), t\right\} \\
& \geq \min \left\{\inf _{y \in\left(\left(a^{\prime} \wedge b \wedge x\right) \vee\left(a \wedge b^{\prime} \wedge x^{\prime}\right)\right)} \mu_{A}(y), \inf _{z \in\left(\left(a^{\prime} \wedge b^{\prime} \wedge x\right) \vee\left(a \wedge b \wedge x^{\prime}\right)\right)} \mu_{A}(z), t\right\} \\
& \geq \min \left\{\inf _{y \in\left(\left(a^{\prime} \wedge b\right) \vee\left(a \wedge b^{\prime}\right)\right)} \mu_{A}(y), \inf _{z \in\left(\left(b^{\prime} \wedge x\right) \vee\left(b \wedge x^{\prime}\right)\right)} \mu_{A}(z), t\right\} \\
& =\min \left\{\inf _{y \in\left(\left(b^{\prime} \wedge x\right) \vee\left(b \wedge x^{\prime}\right)\right)} \mu_{A}(y), t\right\} \\
& =\mu_{[b]_{A}}(x) .
\end{aligned}
$$

That is, $\mu_{[a]_{A}}(x) \geq \mu_{[b]_{A}}(x)$. Also,

$$
\begin{aligned}
\lambda_{[a]_{A}}(x) & =\max \left\{\sup _{y \in\left(\left(a^{\prime} \wedge x\right) \vee\left(a \wedge x^{\prime}\right)\right)} \lambda_{A}(y), t\right\} \\
& =\max \left\{\sup _{y \in\left(\left(\left(a^{\prime} \wedge x\right) \vee\left(a \wedge x^{\prime}\right) \wedge\left(b \vee b^{\prime}\right)\right)\right.} \lambda_{A}(y), t\right\} \\
& =\max \left\{\sup _{y \in\left(\left(a^{\prime} \wedge b \wedge x\right) \vee\left(a \wedge b^{\prime} \wedge x^{\prime}\right) \vee\left(a^{\prime} \wedge b^{\prime} \wedge x\right) \vee\left(a \wedge b \wedge x^{\prime}\right)\right)} \lambda_{A}(y), t\right\} \\
& \leq \max \left\{\sup _{y \in\left(\left(a^{\prime} \wedge b \wedge x\right) \vee\left(a \wedge b^{\prime} \wedge x^{\prime}\right)\right)} \lambda_{A}(y), \sup _{z \in\left(\left(a^{\prime} \wedge b^{\prime} \wedge x\right) \vee\left(a \wedge b \wedge x^{\prime}\right)\right)} \lambda_{A}(z), t\right\} \\
& \leq \max \left\{\sup _{y \in\left(\left(a^{\prime} \wedge b\right) \vee\left(a \wedge b^{\prime}\right)\right)} \lambda_{A}(y), \sup _{z \in\left(\left(b^{\prime} \wedge x\right) \vee\left(b \wedge x^{\prime}\right)\right)} \lambda_{A}(z), t\right\} \\
& =\max \left\{\sup _{y \in\left(\left(b^{\prime} \wedge x\right) \vee\left(b \wedge x^{\prime}\right)\right)} \lambda_{A}(y), t\right\} \\
& =\lambda_{[b]_{A}}(x) .
\end{aligned}
$$

That is, $\lambda_{[a]_{A}}(x) \leq \lambda_{[b]_{A}}(x)$.

By the same method, we have $\mu_{[a]_{A}}(x) \leq \mu_{[b]_{A}}(x)$ and $\lambda_{[a]_{A}}(x) \geq \lambda_{[b]_{A}}(x)$. Hence $\mu_{[a]_{A}}=$ $\mu_{[b]_{A}}$ and $\lambda_{[a]_{A}}(x)=\lambda_{[b]_{A}}(x)$. Therefore $[a]_{A}=[b]_{A}$.

Proposition 5.2 Let $B$ be a Boolean hyperalgebra, $A=\left(\mu_{A}, \lambda_{A}\right)$ be a generalized intuitionistic fuzzy hyperideal of $B, a, b, a_{1}, b_{1} \in B$. Then we have:

(1) If $\mu_{[a]_{A}}=\mu_{\left[a_{1}\right]_{A}}, \mu_{[b]_{A}}=\mu_{\left[b_{1}\right]_{A}}$ and $\lambda_{[a]_{A}}=\lambda_{\left[a_{1}\right]_{A}}, \lambda_{[b]_{A}}=\lambda_{\left[b_{1}\right]_{A}}$, then

$$
\mu_{[a \vee b]_{A}}=\mu_{\left[a_{1} \vee b_{1}\right]_{A}} \text { and } \lambda_{[a \vee b]_{A}}=\lambda_{\left[a a_{1} \vee b_{1}\right]_{A}} \text {, i.e., }[a \vee b]_{A}=\left[a_{1} \vee b_{1}\right]_{A}
$$

(2) If $\mu_{[a]_{A}}=\mu_{\left[a_{1}\right]_{A}}, \mu_{[b]_{A}}=\mu_{\left[b_{1}\right]_{A}}$ and $\lambda_{[a]_{A}}=\lambda_{\left[a_{1}\right]_{A}}, \lambda_{[b]_{A}}=\lambda_{\left[b_{1}\right]_{A}}$, then

$$
\mu_{[a \wedge b]_{A}}=\mu_{\left[a_{1} \wedge b_{1}\right]_{A}} \text { and } \lambda_{[a \wedge b]_{A}}=\lambda_{\left[a_{1} \wedge b_{1}\right]_{A}} \text {, i.e., }[a \wedge b]_{A}=\left[a_{1} \wedge b_{1}\right]_{A} ;
$$

(3) If $\mu_{[a]_{A}}=\mu_{\left[a_{1}\right]_{A}}$ and $\lambda_{[a]_{A}}=\lambda_{\left[a_{1}\right]_{A}}$, then $\mu_{\left[a^{\prime}\right]_{A}}=\mu_{\left[a_{1}^{\prime}\right]_{A}}$ and $\lambda_{\left[a^{\prime}\right]_{A}}=\lambda_{\left[a_{1}^{\prime}\right]_{A}}$, i.e., $\left[a^{\prime}\right]_{A}=\left[a_{1}^{\prime}\right]_{A}$.

Proof (1)

$$
\begin{aligned}
& \inf _{x \in\left(\left((a \vee b)^{\prime} \wedge\left(a_{1} \vee b_{1}\right)\right) \vee\left((a \vee b) \wedge\left(a_{1} \vee b_{1}\right)^{\prime}\right)\right)} \mu_{A}(x) \\
& \quad \geq \inf _{y \in\left(\left(\left(a^{\prime} \wedge b^{\prime}\right) \wedge\left(a_{1} \vee b_{1}\right)\right) \vee\left((a \vee b) \wedge\left(a_{1}^{\prime} \wedge b_{1}^{\prime}\right)\right)\right)} \mu_{A}(y) \\
& \quad \geq \inf _{z \in\left(\left(\left(a^{\prime} \wedge a_{1} \wedge b^{\prime}\right) \vee\left(a \wedge a_{1}^{\prime} \wedge b_{1}^{\prime}\right)\right) \vee\left(\left(a^{\prime} \wedge b^{\prime} \wedge b_{1}^{\prime}\right) \vee\left(a_{1}^{\prime} \wedge b \wedge b_{1}^{\prime}\right)\right)\right)} \mu_{A}(z)
\end{aligned}
$$




$$
\begin{aligned}
& \geq \min \left\{\inf _{w \in\left(\left(\left(a^{\prime} \wedge a_{1} \wedge b^{\prime}\right) \vee\left(a \wedge a_{1}^{\prime} \wedge b_{1}^{\prime}\right)\right)\right)} \mu_{A}(w), \inf _{h \in\left(\left(\left(a^{\prime} \wedge b^{\prime} \wedge b_{1}\right) \vee\left(a_{1}^{\prime} \wedge b \wedge b_{1}^{\prime}\right)\right)\right)} \mu_{A}(h)\right\} \\
& \geq \min \left\{\min \left\{\inf _{p \in\left(\left(a^{\prime} \wedge a_{1}\right) \vee\left(a \wedge a_{1}^{\prime}\right)\right)} \mu_{A}(p), t\right\}, \min \left\{\inf _{q \in\left(\left(b^{\prime} \wedge b_{1}\right) \vee\left(b \wedge b_{1}^{\prime}\right)\right)} \mu_{A}(q), t\right\}\right\} \\
& \geq t .
\end{aligned}
$$

Hence $\mu_{[a \vee b]_{A}}=\mu_{\left[a_{1} \vee b_{1}\right]_{A}}$. Also,

$$
\begin{aligned}
& x \in\left(\left((a \vee b)^{\prime} \wedge\left(a_{1} \vee b_{1}\right)\right) \vee\left((a \vee b) \wedge\left(a_{1} \vee b_{1}\right)^{\prime}\right)\right) \\
& \leq \sup _{y \in\left(\left(\left(a^{\prime} \wedge b^{\prime}\right) \wedge\left(a_{1} \vee b_{1}\right)\right) \vee\left((a \vee b) \wedge\left(a_{1}^{\prime} \wedge b_{1}^{\prime}\right)\right)\right)} \lambda_{A}(y) \\
& \leq \sup _{z \in\left(\left(\left(a^{\prime} \wedge a_{1} \wedge b^{\prime}\right) \vee\left(a \wedge a_{1}^{\prime} \wedge b_{1}^{\prime}\right)\right) \vee\left(\left(a^{\prime} \wedge b^{\prime} \wedge b_{1}^{\prime}\right) \vee\left(a_{1}^{\prime} \wedge b \wedge b_{1}^{\prime}\right)\right)\right)} \lambda_{A}(z) \\
& \leq \max \left\{\sup _{w \in\left(\left(\left(a^{\prime} \wedge a_{1} \wedge b^{\prime}\right) \vee\left(a \wedge a_{1}^{\prime} \wedge b_{1}^{\prime}\right)\right)\right)} \lambda_{A}(w), \sup _{h \in\left(\left(\left(a^{\prime} \wedge b^{\prime} \wedge b_{1}\right) \vee\left(a_{1}^{\prime} \wedge b \wedge b_{1}^{\prime}\right)\right)\right)} \lambda_{A}(h)\right\} \\
& \quad \leq \max \left\{\max \left\{\sup _{p \in\left(\left(a^{\prime} \wedge a_{1}\right) \vee\left(a \wedge a_{1}^{\prime}\right)\right)} \lambda_{A}(p), t\right\}, \max \left\{\sup _{q \in\left(\left(b^{\prime} \wedge b_{1}\right) \vee\left(b \wedge b_{1}^{\prime}\right)\right)} \lambda_{A}(q), t\right\}\right\} \\
& \leq t .
\end{aligned}
$$

Hence $\lambda_{[a \vee b]_{A}}=\lambda_{\left[a_{1} \vee b_{1}\right]_{A}}$. Therefore $[a \vee b]_{A}=\left[a_{1} \vee b_{1}\right]_{A}$.

Similarly we can prove (2) and (3).

Let $A=\left(\mu_{A}, \lambda_{A}\right)$ be a generalized intuitionistic fuzzy hyperideal of the Boolean hyperalgebra $B$. The hyperoperations $\vee, \wedge$ and the operation ' of $B / A$ are defined as follows:

For all $[a]_{A}=\left(\mu_{[a]_{A}}, \lambda_{[a]_{A}}\right),[b]_{A}=\left(\mu_{[b]_{A}}, \lambda_{[b]_{A}}\right) \in B / A$, then

$$
\begin{aligned}
& {[a]_{A} \vee[b]_{A}=\left\{\left\langle x, \mu_{[a \vee b]_{A}}(x), \lambda_{[a \vee b]_{A}}(x)\right\rangle \mid x \in B\right\}} \\
& {[a]_{A} \wedge[b]_{A}=\left\{\left\langle x, \mu_{[a \wedge b]_{A}}(x), \lambda_{[a \wedge b]_{A}}(x)\right\rangle \mid x \in B\right\}} \\
& \left([a]_{A}\right)^{\prime}=\left\{\left\langle x, \mu_{\left([a]_{A}\right)^{\prime}}(x), \lambda_{\left([a]_{A}\right)^{\prime}}(x)\right\rangle \mid x \in B\right\},
\end{aligned}
$$

where

$$
\begin{aligned}
& \mu_{[a \vee b]_{A}}=\left\{\mu_{[x]_{A}}: x \in a \vee b\right\} \text { and } \lambda_{[a \vee b]_{A}}=\left\{\lambda_{[x]_{A}}: x \in a \vee b\right\} ; \\
& \mu_{[a \wedge b]_{A}}=\left\{\mu_{[x]_{A}}: x \in a \wedge b\right\} \text { and } \lambda_{[a \wedge b]_{A}}=\left\{\lambda_{[x]_{A}}: x \in a \wedge b\right\} ; \\
& \mu_{\left([a]_{A}\right)^{\prime}}=\mu_{\left[a^{\prime}\right]_{A}} \text { and } \lambda_{\left([a]_{A}\right)^{\prime}}=\lambda_{\left[a^{\prime}\right]_{A}} .
\end{aligned}
$$

By Proposition 5.2, the above hyperoperations and operation are reasonable.

Proposition 5.3 Let $B$ be a Boolean hyperalgebra, $A=\left(\mu_{A}, \lambda_{A}\right)$ be a generalized intuitionistic fuzzy proper hyperideal of $B$. Then $B / A=\left\{B / A ; \vee, \wedge,,^{\prime},[0]_{A},[1]_{A}\right\}$ is a Boolean hyperalgebra.

Proof It is easy and omitted. 
Let $B$ be a Boolean hyperalgebra, $A=\left(\mu_{A}, \lambda_{A}\right)$ be a generalized intuitionistic fuzzy subhyperalgebra of $B, C=\left(\mu_{C}, \lambda_{C}\right)$ be a generalized intuitionistic fuzzy proper hyperideal of $B$. We define an intuitionistic fuzzy subset of $A / C$ as follows:

$$
A / C=\left\{\left\langle x, \mu_{A / C}(x), \lambda_{A / C}(x)|| x \in B\right\}\right.
$$

where $\mu_{A / C}: B / C \rightarrow[0,1]$

$$
\mu_{A / C}\left([a]_{C}\right)=\sup _{[x]_{C} \subseteq[a]_{C}} \mu_{C}(x), \quad \forall[a]_{C} \in B / C,
$$

and $\lambda_{A / C}: B / C \rightarrow[0,1]$

$$
\lambda_{A / C}\left([a]_{C}\right)=\inf _{[x]_{C} \subseteq[a]_{C}} \lambda_{C}(x), \quad \forall[a]_{C} \in B / C .
$$

Proposition 5.4 Let $B$ be a Boolean hyperalgebra, $A=\left(\mu_{A}, \lambda_{A}\right)$ be a generalized fuzzy subhyperalgebra of $B, C=\left(\mu_{C}, \lambda_{C}\right)$ be a generalized intuitionistic fuzzy proper hyperideal of $B$, then $A / C$ is a generalized intuitionistic fuzzy subhyperalgebra of $B / C$.

Proof (1) $\forall[a]_{C},[b]_{C} \in B / C$, we have

$$
\begin{aligned}
& \inf _{[x]_{C} \subseteq\left([a]_{C} \vee[b]_{C}\right)} \mu_{A / C}\left([x]_{C}\right) \\
& =\inf _{[x]_{C} \subseteq\left([a \vee b]_{C}\right)} \mu_{A / C}\left([x]_{C}\right)=\inf _{[x]_{C} \subseteq\left([a \vee b]_{C}\right)} \sup _{[y]_{C} \subseteq[x]_{C}} \mu_{C}(y) \\
& \geq \sup _{[p]_{C} \subseteq[a]_{C},[q]_{C} \subseteq[b]_{C}, z \in p \vee q} \mu_{C}(z) \geq \sup _{[p]_{C} \subseteq[a]_{C},[q]_{C} \subseteq[b]_{C}} \min \left\{\mu_{C}(p), \mu_{C}(q), t\right\} \\
& =\min \left\{\left(\sup _{[p]_{C} \subseteq[a]_{C}} \mu_{C}(p)\right),\left(\sup _{[q]_{C} \subseteq[b]_{C}} \mu_{C}(q)\right), t\right\}=\min \left\{\mu_{A / C}\left([a]_{C}\right), \mu_{A / C}\left([b]_{C}\right), t\right\},
\end{aligned}
$$

and $\forall[a]_{C},[b]_{C} \in B / C$, we have

$$
\begin{aligned}
& \sup _{[x]_{C} \subseteq\left([a]_{C} \vee[b]_{C}\right)} \lambda_{A / C}\left([x]_{C}\right) \\
& =\sup _{[x]_{C} \subseteq\left([a \vee b]_{C}\right)} \lambda_{A / C}\left([x]_{C}\right)=\sup _{[x]_{C} \subseteq\left([a \vee b]_{C}\right)} \inf _{C} \subseteq[x]_{C} \lambda_{C}(y) \\
& \leq_{[p]_{C} \subseteq[a]_{C},[q]_{C} \subseteq[b]_{C}, z \in p \vee q} \lambda_{C}(z) \leq \inf _{[p]_{C} \subseteq[a]_{C},[q]_{C} \subseteq[b]_{C}} \max \left\{\lambda_{C}(p), \lambda_{C}(q), t\right\} \\
& =\max \left\{\left(\inf _{[p]_{C} \subseteq[a]_{C}} \lambda_{C}(p)\right),\left(\inf _{[q]_{C} \subseteq[b]_{C}} \mu_{C}(q)\right), t\right\}=\max \left\{\lambda_{A / C}\left([a]_{C}\right), \lambda_{A / C}\left([b]_{C}\right), t\right\} .
\end{aligned}
$$

(2) For all $[a]_{C} \in B / C$,

$$
\begin{aligned}
\mu_{A / C}\left(\left([a]_{C}\right)^{\prime}\right) & =\mu_{A / C}\left(\left[a^{\prime}\right]_{C}\right) \\
& =\sup _{\left[x^{\prime}\right]_{C} \subseteq\left[a^{\prime}\right]_{C}} \mu_{C}\left(x^{\prime}\right) \\
& \geq \sup _{\left([x]_{C}\right)^{\prime} \subseteq\left([a]_{C}\right)^{\prime}} \min \left\{\mu_{C}(x), t\right\}
\end{aligned}
$$




$$
\begin{aligned}
& =\min \left\{\left(\sup _{[x]_{C} \subseteq[a]_{C}} \mu_{C}(x)\right), t\right\} \\
& =\min \left\{\mu_{A / C}\left([a]_{C}\right), t\right\},
\end{aligned}
$$

and

$$
\begin{aligned}
\lambda_{A / C}\left(\left([a]_{C}\right)^{\prime}\right) & =\lambda_{A / C}\left(\left[a^{\prime}\right]_{C}\right) \\
& =\inf _{\left[x^{\prime}\right]_{C} \subseteq\left[a^{\prime}\right]_{C}} \lambda_{C}\left(x^{\prime}\right) \\
& \leq \inf _{\left([x]_{C}\right)^{\prime} \subseteq\left([a]_{C}\right)^{\prime}} \max \left\{\lambda_{C}(x), t\right\} \\
& =\max \left\{\left(\inf _{[x]_{C} \subseteq[a]_{C}} \lambda_{C}(x)\right), t\right\} \\
& =\max \left\{\lambda_{A / C}\left([a]_{C}\right), t\right\} .
\end{aligned}
$$

Hence $A / C$ is a generalized intuitionistic fuzzy subhyperalgebra of $B / C$.

Definition 5.5 Let $B$ be a Boolean hyperalgebra, $A=\left(\mu_{A}, \lambda_{A}\right)$ be a generalized intuitionistic fuzzy subhyperalgebra of $B, C=\left(\mu_{C}, \lambda_{C}\right)$ be a generalized intuitionistic fuzzy proper hyperideal of $B$, then $A / C$ is called generalized intuitionistic fuzzy quotient Boolean hyperalgebra of $A$ with respect to $C$.

Definition 5.6 Let $B, B^{\prime}$ be two Boolean hyperalgebras, $f: B \rightarrow B^{\prime}$ be a Boolean hyperalgebra homomorphism, $A=\left(\mu_{A}, \lambda_{A}\right), C=\left(\mu_{C}, \lambda_{C}\right)$ be generalized intuitionistic fuzzy subhyperalgebras of $B$ and $B^{\prime}$, respectively. If $f(A)=C$, then we say $A$ is homomorphic to $C$, which is denoted as $A \backsim C$.

Proposition 5.7 Let $B$ be a Boolean hyperalgebra, $A=\left(\mu_{A}, \lambda_{A}\right)$ be a generalized intuitionistic fuzzy subhyperalgebra of $B, C=\left(\mu_{C}, \lambda_{C}\right)$ be a generalized intuitionistic fuzzy proper hyperideal of $B$. Then $A \backsim A / C$.

Proof Let $f: B \rightarrow B / C, f(x)=[x]_{C}, \forall x \in B$. For all $a, b \in B$, we have

$$
\begin{aligned}
& f(a \vee b)=[a \vee b]_{C}=[a]_{C} \vee[b]_{C}=f(a) \vee f(b), \\
& f(a \wedge b)=[a \wedge b]_{C}=[a]_{C} \wedge[b]_{C}=f(a) \wedge f(b), \\
& f\left(a^{\prime}\right)=\left[a^{\prime}\right]_{C}=\left([a]_{C}\right)^{\prime}=(f(a))^{\prime} .
\end{aligned}
$$

Hence $f$ is a Boolean hyperalgebra homomorphism.

For all $[a]_{C} \in B / C$, we have

$$
\begin{aligned}
f\left(\mu_{A / C}\right)\left([a]_{C}\right) & =\sup _{f(x) \subseteq[a]_{C}} \mu_{C}(x) \\
& =\sup _{[x]_{C} \subseteq[a]_{C}} \mu_{C}(x) \\
& =\mu_{A / C}\left([a]_{C}\right),
\end{aligned}
$$


and

$$
\begin{aligned}
f^{*}\left(\lambda_{A / C}\right)\left([a]_{C}\right) & =\inf _{f(x) \subseteq[a]_{C}} \lambda_{C}(x) \\
& =\inf _{[x]_{C} \subseteq[a]_{C}} \lambda_{C}(x) \\
& =\lambda_{A / C}\left([a]_{C}\right) .
\end{aligned}
$$

Hence $A \backsim A / C$.

\section{Competing interests}

The authors declare that they have no competing interests.

\section{Authors' contributions}

All authors read and approved the final manuscript.

\section{Author details}

1 Department of Mathematics \& Computer Science, Faculty of Natural Sciences, University of Gjirokastra, Gjirokastra, Albania. ${ }^{2}$ Department of Mathematics, Yildiz Technical University, Istanbul, 81270, Turkey. ${ }^{3}$ Department of Mathematics, Yazd University, Yazd, Iran.

Received: 24 June 2013 Accepted: 30 September 2013 Published: 08 Nov 2013

\section{References}

1. Marty, F: Sur une generalization de la notion de group. In: 8th Congres Math. Scandinaves, Stockholm, pp. 45-49 (1934)

2. Corsini, P: Prolegomena of Hypergroup Theory, 2nd edn. Aviani Editor, Tricesimo (1993)

3. Corsini, P, Leoreanu-Fotea, V: Applications of Hyperstructure Theory, Advances in Mathematics. Kluwer Academic, Dordrecht (2003)

4. Davvaz, B, Leoreanu-Fotea, V: Hyperring Theory and Applications. International Academic Press, Palm Harber (2007)

5. Vougiouklis, T: Hyperstructures and Their Representations, vol. 115. Hadronic Press, Palm Harber (1994)

6. Konstantinidou, M, Mittas, J: An introduction to the theory of hyperlattices. Math. Balk. 7, 187-193 (1977)

7. Serafimidis, K, Kehagias, $A$, Konstantinidou, M: The structure of the $(P, Q)$-superlattice and order related properties. Ital. J. Pure Appl. Math. 15, 133-150 (2004)

8. Serafimidis, K, Kehagias, A: Some representation results for ( $P, Q)$-superlattices. Ital. J. Pure Appl. Math. 15, 151-164 (2004)

9. Zadeh, LA: Fuzzy sets. Inf. Control 8, 338-353 (1965)

10. Rosenfeld, A: Fuzzy groups. J. Math. Anal. Appl. 35(3), 512-517 (1971)

11. Abdullah, S, Aslam, M, Khan, TA, Naeem, M: A new type of fuzzy normal subgroup and fuzzy coset. J. Intell. Fuzzy Syst. 25, 37-47 (2013)

12. Chen, DG, Li, SY: Fuzzy factor rings. Fuzzy Sets Syst. 94, 125-127 (1998)

13. Gu, WX, Lu, T: Fuzzy algebras over fuzzy fields redefined. Fuzzy Sets Syst. 53, 105-107 (1993)

14. Nanda, S: Fuzzy algebras over a fuzzy fields. Fuzzy Sets Syst. 37, 99-103 (1990)

15. Yuan, XH, Zhang, C, Ren, YH: Generalized groups and many-valued implications. Fuzzy Sets Syst. 138, $205-211$ (2003)

16. Kuraoka, T, Nobuaki, K: On fuzzy quotient-rings induced by fuzzy ideals. Fuzzy Sets Syst. 47, 381-386 (1992)

17. Davvaz, B: A survey of fuzzy algebraic hyperstructures. Algebras Groups Geom. 27, 37-62 (2010)

18. Corsini, P, Leoreanu-Fotea, V: Fuzzy sets and join spaces associated with rough sets. Rend. Circ. Mat. Palermo 2(51) 527-536 (2002)

19. Atanassov, K: Intuitionistic fuzzy sets. Central Tech. Library, Bulgarian Academy Science, Sofia, Bulgaria, Rep. No. 1697/84 (1983)

20. Atanassov, K: Intuitionistic Fuzzy Sets, Theory and Applications. Studies in Fuzziness and Soft Computing, vol. 35. Physica-Verlag, Heidelberg (1999)

21. Abdullah, S, Davvaz, B, Aslam, M: $(\alpha, \beta)$-Intuitionistic fuzzy ideals in hemirings. Comput. Math. Appl. 62(8), $3077-3090$ (2011)

22. Abdullah, S, Aslam, $\mathrm{M}$, Hedayati, $\mathrm{H}$ : Interval valued $(\alpha, \beta)$-intuitionistic fuzzy ideals in hemirings. J. Intell. Fuzzy Syst. doi:10.3233/IFS-130954

23. Ahmad, A, Aslam, M, Abdullah, S: Interval valued $(\alpha, \beta)$-fuzzy hyperideals of semihyperring. Sci. Bull. "Politeh." Univ. Buchar., Ser. A, Appl. Math. Phys. 75(2), 69-86 (2013)

24. Davvaz, B, Dudek, WA, Jun, YB: Intuitionistic fuzzy Hv-submodules. Inf. Sci. 176, 285-300 (2006)

25. Dudek, WA, Davvaz, B, Jun, YB: On intuitionistic fuzzy subhyperquasi-groups of hyperquasigroups. Inf. Sci. 170 251-262 (2005)

26. Hila, K, Abdullah, S: A study on intuitionistic fuzzy sets in $\Gamma$-semihypergroups. J. Intell. Fuzzy Syst. doi:10.3233/IFS-130849

27. Sun, SQ: Fuzzy subalgebras and fuzzy ideals of Boolean algebra. Fuzzy Syst. Math. 20(1), 90-94 (2006)

28. Sun, SQ, Gui, WX: Fuzzy congruence relations on Boolean algebra. Fuzzy Syst. Math. 19(4), 34-38 (2005) 
29. Sun, SQ: Generalized fuzzy subalgebras of Boolean algebras. In: Proceedings of the International Workshop on Information Security and Application (IWISA 2009), Qingdao, China, November 21-22 (2009)

30. Liu, X, Ye, J: Fuzzy Boolean algebra and its properties, 53-61. Preprint

31. Liu, XH, Yu, Y: The nature of finite fuzzy Boolean algebras. Fuzzy Math. 6(4), 21-24 (1986)

32. Mittas, J, Konstantinidou, M: Introduction a' l'hyperalgebre de Boole. Math. Balk. 51, 314-320 (1976)

33. Konstantinidou, MK, Synefaki, Al: A strong Boolean hyperalgebra of Boolean functions. Ital. J. Pure Appl. Math. 2, 9-18 (1997)

34. Procesi Ciampi, R, Rota, R: Stone's representation theorem for Boolean hyperalgebras. In: Proc. Fourth Int. Congr. AHA Xanthi (1990)

35. Procesi Ciampi, R: Stone's representation theorem for Boolean hyperalgebras topological version. Discrete Math. 155, 211-214 (1996)

36. Lu, JJ, Zhu, YQ, Sun, GT: Boolean Algebra. Shandong Education Press, Jinan (1982)

37. Atanassov, K: Intuitionistic fuzzy sets. Fuzzy Sets Syst. 20, 87-96 (1986)

38. Atanassov, K: New operations defined over the intuitionistic fuzzy sets. Fuzzy Sets Syst. 61, 137-142 (1994)

10.1186/1029-242X-2013-501

Cite this article as: Hila et al.: On generalized intuitionistic fuzzy subhyperalgebras of Boolean hyperalgebras. Journal of Inequalities and Applications 2013, 2013:501

\section{Submit your manuscript to a SpringerOpen ${ }^{\ominus}$ journal and benefit from:}

- Convenient online submission

- Rigorous peer review

- Immediate publication on acceptance

- Open access: articles freely available online

- High visibility within the field

- Retaining the copyright to your article 